\title{
Rationalizing combination therapies
}

\author{
A growing number of clinical trials on combination therapies raises the question of to what degree they may be \\ redundant. Systems biology and hypothesis-driven preclinical studies could help to identify the most promising \\ candidates for clinical trials, and also offer new insights into the biological mechanisms that underlie drug synergies.
}

$\mathbf{T}$ here are more than 10,000 ongoing clinical trials currently registered in the US alone investigating combination therapies for cancer, infectious diseases, and metabolic, cardiovascular, autoimmune, and neurological disorders. There are even more preclinicalresearch articles on drug combinations. Yet, these numbers are quite modest relative to all potential combinations that could be tested. This raises the questions of what strategies are best for selecting candidates for combination testing preclinically, and how the success of the results is being judged for transition to the clinic.

Quite often, combination treatments are compared to single drugs and are considered successful if they produce a better response together than the individual drugs by themselves. But because many drug combinations can have additive or even synergistic effects, it is the degree of synergy that becomes the essential question. Answering it requires benchmarking the two-drug combination against not only the single treatments, but also the best of previously reported drug combinations. Although such multiple comparisons would be difficult to incorporate into clinical studies, side-by-side comparisons of drug combinations in preclinical research, for example, by high-throughput screening, could help to select better candidates for clinical trials. Testing multiple combinations and analyzing the degree of synergy in a screen can reveal patterns suggestive of the mechanisms behind drug interactions. Understanding the mechanism of drug synergy, rather than simply knowing which drugs to combine, enables further optimization of beneficial drug interactions and can offer important insights into the underlying biology.

Rational design of preclinical testing can be of further help. One rationale behind the success of combination therapies lies in their ability to effectively influence biological regulatory circuits, which have built-in redundancies that make them resistant to single perturbations, as well as feedback mechanisms that enable them to adapt to changes in the environment and maintain homeostasis. By targeting multiple components of these circuits at once, combination therapies might produce a stronger treatment for a disease than individual treatments, according to theoretical modeling (eLife 2, e00747, 2013).

Modeling disease-associated regulatory circuits using systems-biology tools offers one educated design strategy for testing combination therapies. This approach requires a computational model that describes the molecular and cellular regulatory circuits of the disease state, steady state, and transitions between them. This type of modeling enables researchers to make important predictions, such as how many pathways need to be targeted by drugs to overcome or prevent resistance, and the minimum number of drugs required for a combination therapy to achieve a good outcome. Is a triple combination of drugs always better than a double? Are quadruple-drug combinations better than triple? Systems biology can answer this question on the basis of the signaling-network architecture.

Modeling is a useful approach for designing drugs that target wellunderstood disease states, such as deregulated molecular pathways controlling cell survival, proliferation, and metabolism. However, many diseases involve the deregulation of higher-order physiological circuits composed of multiple cell types, tissues, and organs. The complexity of these interactions exceeds the current level of mechanistic understanding required to build accurate network models. In such cases, rational choices of combination drugs can be made according to the physiologybased hypothesis, as illustrated by recent advances in immunotherapies. Blockade of the inhibitory immune receptor CTLA-4 promotes antitumor immune responses, but compensatory upregulation of the $\mathrm{PD}-1$ receptor and its ligands PD-L1/2 often leads to resistance. A combination of CTLA- 4 and PD- 1 activities overcomes this resistance and multiplies the efficacy of single treatments (Am. J. Clin. Oncol. 39, 98-106, 2016).

Even when the basic concept explaining drug synergy seems clear, the criteria for clinical success require further study. For example, both tremelimumab (anti-CTLA-4) and durvalumab (anti-PD-L1) monoclonal antibodies have shown efficacy as single checkpoint-blockade immunotherapies for several cancer types, as have analogous reagents developed by others-ipilimumab targeting CTLA-4, and nivolumab targeting PD-1. Moreover, ipilimumab and nivolumab combination therapies have shown synergy in clinical trials, including those investigating non-small-cell lung cancer. Yet, the combination of tremelimumab and durvalumab in the MYSTIC trial has not provided any additional clinical benefit to individuals with non-small-cell lung cancer, as announced in July 2017. The difference in efficacy could be due to the drug targeting PD-L1 rather than PD-1 or to the use of different antibody isotype backbones. These possibilities can be tested preclinically and should guide the next round of therapeutics.

Testing proprietary drugs such as the ones used in the MYSTIC trial is associated with regulatory, financial, and logistical hurdles (Nat. Med. 21, 415, 2015; N. Engl. J. Med. 364, 985-987; 2011), which add up when more than one drug is to be studied. To facilitate combination-treatment testing, in January, the National Cancer Institute (NCI) launched the NCI Formulary program, which provides researchers with access to both experimental cancer therapeutics and those recently approved by the US Food and Drug Administration. Its growing list of drugs available for preclinical and clinical combination research includes all four of the immune-checkpoint inhibitors discussed above.

Combination therapies are becoming a part of routine evaluation in the development of every drug and treatment for every disease. To make these efforts worthwhile, intelligent study design and defined criteria of success are needed to benchmark preclinical discoveries and thus make for more successful clinical trials. 